\title{
Inhalt, Vol. 12, No. 5, 1989
}

Contents

Impressum 198

Editorial 200

Queißer, W.; Flechtner, H.; Heim, M.E. (Mannheim); Kabelitz, K. (Homburg/Saar); Maßner, B. (Frankenthal); Edler, L. (Heidelberg)

Dosiseskalation und -modifikation von 4-Epidoxorubicin

in der Kombinationschemotherapie (FEM II) des fortge-

schrittenen Magenkarzinoms 202

Hinweise für Autoren 208

Günczler, P.; Ogris, E.; Maca, S.; Danmayr, E.

(Wien/Österreich)

Tumormarker bei Mammakarzinom: Zur diagnostischen

Wertigkeit von Serumbestimmungen bei klinischer Tumor-

freiheit (NED) und manifester Erkrankung 209

Meden, H.; Rath, W.; Teichmann, A.; Kuhn, W.

(Göttingen)

Serumspiegel des Tumormarkers CA125 vor der Second-

look-Laparotomie beim primären epithelialen Ovarial-

karzinom in Relation zu intraoperativen Befunden .... 217

Buchbesprechungen ' 221

Informationen für die Klinik 224

Hausmaninger, H. (Salzburg/Österreich); Lehnert, M.; Samonígg, H. (Graz/Österreich); Steger, G.; Sevelda, P.; KieПzer, R. (Wien/Österreich); Míchlmayr, G.; Hehen-warter, W.; Fridrik, M.

(Linz/Österreich); Schiller, L. (Vöcklabruck/Österreich); Manfreda, D. (Klagenfurt/ Österreich);

Haídínger, R. (Steyr/Österreich); Neiss, A. (Innsbruck/Österreich)

Vindesin-Epirubicin (VE) versus Vindesin-Mitoxantron

(VM) beim metastasierenden Mammakarzinom 225

Hallek, M.; Haen, E.; Emmerich, B. (München);

Lévi, F. (Villejuif/Frankreich)

Bedeutung der Chronopharmakologie für die Onkologie 230

Manegold, C; Biilzebruck, H.; Drings, P.; Vogt-Moykopf, I. (Heidelberg)

Prognostische Faktoren beim kleinzelligen Bronchial-

karzinom 240

AIO-Mitteilungen $\quad 246$

Imprint198

Editorial 200

Queißer, W.; Flechtner, H.; Heim, M. E. (Mannheim); Kabelitz, K. (Homburg/Saar); Maßner, B. (Frankenthal); Edler, L. (Heidelberg) 
Dose Escalation and Split Courses of 4-Epidoxorubicin in Combination Chemotherapy (FEM II) of Advanced Gas tric Carcinoma. A Phase-II Trial of the 'ChemotherapiegruppegastrointestinalerTumoren(CGT)' 202 Instructions to Authors 208 Günczler, P.; Ogris, E.; Maca, S.; Danmayr, E. (Vienna/Austria) Clinical Value of Tumor Marker Determinations in Breast Cancer: Analysis of Serum Levels in Tumor-Bear ing and Disease-Free Patients 209 Meden, H.; Rath, W.; Teichmann, A.; Kuhn, W. (Göttingen) Preoperative Serum CA125 Levels in Patients with Pri mary Epithelial Ovarian Cancer in Relation to Findings at Second-Look Laparotomy 217

Book Reviews 221

Clinical Information 224

Hausmaninger, H. (Salzburg/Austria); Lehnert, M.; Samonigg, H. (Graz/Austria); Steger, G.; Sevelda, P.; Kienzer, R. (Vienna/Austria); Michlmayr, G.; Hehen-warter, W.; Fridrik, M. (Linz/Austria); Schiller, L. (Vöcklabruck/Austria); Manfreda, D. (Klagenfurt/ Austria);

Haidínger, R. (Steyr/Austria); Neiss, A. (Innsbruck/Austri a)

Vindesine-Epirubicin versus Vindesine-Mitoxantrone in

Metastatic Breast Cancer 225

Hallek, M.; Haen, E.; Emmerich, B. (Munich);

Lévi, F. (Villejuif/France)

Importance of Chronopharmacology in Oncology 230

Manegold, C; Biilzebruck, H.; Drings, P.;

Vogt-Moykopf, L (Heidelberg)

Prognostic Factors in Small Cell Lung Cancer $\quad 240$

AIO Reports . 246

Bibliographischer Hinweis: Inhaltsverzeichnisse dieser Zeitschrift erscheinen regelmäßig in current contents ${ }^{\circledR}$ sowie in anderen bibliographischen Diensten. 